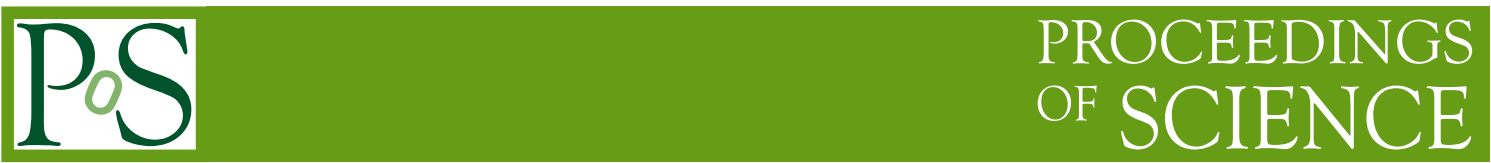

\title{
UHE neutrinos: from standard astrophysics to new physics
}

\author{
V. Berezinsky \\ INFN. Laboratori Nazionali del Gran Sasso, 67010 Assergi (AQ) Italy
}

\begin{abstract}
Neutrinos with $E>10^{17} \mathrm{eV}$ are produced by ultra-high energy cosmic rays (UHECR) interacting with CMB and EBL photons (cosmogenic neutrinos) and by top-down sources, such as topological defects (TDs) and superheavy dark matter (SHDM). Cosmogenic neutrinos are reliably predicted and their fluxes can be numerically evaluated using the observed flux of UHECR. The lower limit for the flux is obtained for the case of pure proton composition of the observed UHECR. The rigorous upper limit for cosmogenic neutrino flux also exists. The maximum neutrino energy is determined by maximum energy of acceleration, which at least for the shock acceleration is expected not to exceed $10^{21}-10^{22} \mathrm{eV}$. The top-down sources provide neutrinos with energies a few orders of magnitude higher, and this can be considered as a signature of these models. UHE neutrino fluxes from TDs, SHDM and Mirror Matter are discussed.
\end{abstract}

Quarks, Strings and the Cosmos - Hector Rubinstein Memorial Symposium

August 09-11, 2010

AlbaNova (Stockholm) Sweden 


\section{Introduction}

Hector loved the right physics passionately, and hated the wrong one violently. For such strong feelings one must distinguish between them. Hector really did. When I first told Hector about cosmic neutrinos with energies above $10^{19} \mathrm{eV}$, he exclaimed: 'We will detect them here in Swedish lakes! There is no water in world more transparent!' Neutrinos were never detected in transparent Swedish water, but UHE neutrino astronomy started its development in Sweden thanks to Hector. Swedish team became one of most important collaborator in Amanda and IceCube. Before Hector thought about collaboration between USSR and Sweden. When we invited him to Moscow for scientific visit, Hector asked me whether he can discuss the USSR-Sweden collaboration with the President of the USSR Academy of Sciences. I laughed: one should ask for it at least a month in advance. However, Vitalii Lazarevich Ginzburg, with whom I had at that time close scientific contacts, immediately got 20 min appointment for himself, Moisei Alexandrovich Markov, the head of Nuclear Department of the Academy, Hector and me. In the end of the charming and quite useless discussion, Hector suddenly changed the subject raising the problem of scientific publications and their evaluation. In a few sharp and clear sentences he outlined the problem, and the President, Gurii Ivanovich Marchuk, has just blazed up with interest. After not less than half an hour discussion of this subject we left the President office, in fact the luxurious palace hall, and entered the equally luxurious reception hall, full of famous visitors, waiting impatiently for appointments.

UHE neutrino astronomy at energies above $10^{17} \mathrm{eV}$ is based on new, very efficient methods of neutrino detection and on exiting theories for their production. The most interesting range of this astronomy covers tremendously high energies above $10^{19}-10^{20} \mathrm{eV}$. In fact, this energy scale gives only the low-energy threshold, where the new observational methods, such as space-based observations of fluorescent light as well as radio and acoustic methods start to operate. These methods allow to control the great area and to detect the tiny fluxes of neutrinos. For example the exposure of the space detector JEM-EUSO [1] is planned to reach $6 \times 10^{5} \mathrm{~km}^{2} \mathrm{yr}$ sr, and in project LORD [2] (detection of radio signal from lunar regolith) $1 \times 10^{6} \mathrm{~km}^{2} \mathrm{yr}$ sr. The upper limit obtained on detector ANITA [3] already excludes some models for UHE neutrinos. The upper limits obtained by different detectors are presented in Fig. 1.

The prospects for UHE neutrino astronomy appeared in 1960s soon after prediction of the GZK cutoff [4]. It has been realized [5] that proton interaction with CMB photons at large redshifts in case of cosmological evolution of the sources can produce UHE neutrino fluxes much higher than the observed UHECR flux. At present there are many calculations for fluxes of these cosmogenic neutrinos based on the various models for the observed UHECR flux (see e.g. [6] -[13]). The common features of these calculations are normalization to the observed UHECR flux and using the assumptions on cosmological evolution of the sources and maximum energy of acceleration $E_{\text {acc }}^{\max }$.

From theoretical point of view UHE neutrino astronomy is characterized by well balanced program of observation of cosmogenic neutrinos, based on very conservative assumptions, and neutrinos from top-down scenarios, where neutrinos are produced in the decays or annihilation of very heavy particles. 
The top-down scenarios naturally provide neutrinos with energies higher and much higher than $1 \times 10^{20} \mathrm{eV}$. The idea common to many mechanisms is given by the existence of superheavy particles with very large masses up to the GUT scale $\sim 10^{16} \mathrm{GeV}$. Such particles can be produced by Topological Defects (TDs) (see $[14,15]$ for the reviews). They then rapidly decay and produce a parton cascade, which is terminated by production of pions and other hadrons. Neutrinos are produced in hadron decays.

The production of unstable superheavy particles - the constituent fields of TD - is a very common feature of the TDs. For example, one of the simplest TDs, cosmic strings, produce superheavy particles by many mechanisms: collapse of the string loops, self-intersections, annihilation of cusps, production and annihilation of tiny loops. However, in most of these cases the rate of superheavy particle production and neutrino fluxes are very low. There is one particular case of powerful radiation of very high energy neutrinos by ordinary and superconducting strings. In the string loops there is a point, cusp, which periodically obtains the velocity of light. The loop segment around the cusp moves with very large Lorentz factor $\Gamma$. When a superheavy particle with mass $m_{X}$ is emitted through such segment, its energy $\Gamma m_{X}$ can exceed even Planck energy.

The following TDs are in principle of interest for UHECR and UHE neutrinos [15]: monopoles, ordinary strings, monopoles connected by strings (each monopole is attached to several strings), necklaces (each monopole is attached to two string) and superconducting strings.

In the simple GUT models the superheavy particles are very short-lived. However, in more complicated models the superheavy particles can be long-lived with lifetime exceeding the age of the universe $t_{0}$. Such particles can compose Dark Matter (DM). The Superheavy Dark Matter Model (SHDM) can provide detectable UHE neutrino flux.

The signature of all afore-mentioned top-down models is a natural production of UHE neutrinos with energy much higher than $10^{20} \mathrm{eV}$.

\section{Cascade upper limit on diffuse neutrino flux}

The e-m cascade upper bound puts the rigorous upper limit on UHE neutrino flux [16, 17]. This limit, in contrast to WB upper limit [18], is valid for both accelerator and non-accelerator neutrinos. The production of neutrinos in all these scenarios is accompanied by production of high energy photons and electrons from decay of pions. Colliding with low-energy target photons, a primary photon or electron produces e-m cascade due to reactions $\gamma+\gamma_{\operatorname{tar}} \rightarrow e^{+}+e^{-}, e+\gamma_{\operatorname{tar}} \rightarrow e^{\prime}+\gamma^{\prime}$, etc. The cascade spectrum in its high-energy part is proportional to $E^{-2}$, which is very close to the EGRET observations in the range $10 \mathrm{MeV}-100 \mathrm{GeV}$ [19]. The observed energy density in this range is $\omega_{\text {EGRET }} \approx(2-3) \times 10^{-6} \mathrm{eV} / \mathrm{cm}^{3}$. The cascade energy density must be $\omega_{\text {cas }} \leq \omega_{\text {EGRET }}$, and it limits diffuse neutrino flux. The situation has dramatically changed with the new data of FermiLAT [20] on the flux and spectrum of diffuse extragalactic gamma-radiation. In comparison with EGRET this flux is lower and spectrum is steeper $\left(\propto E^{-2.4}\right)$. It results in stronger upper limit on the cascade energy density $\omega_{\text {cas }} \leq 5.8 \times 10^{-7} \mathrm{eV} / \mathrm{cm}^{3}$ [21], which severely diminishes the allowed UHE neutrino fluxes [21, 22]. The maximally allowed cascade energy density $\omega_{\mathrm{cas}}^{\max } \approx 5.8 \times 10^{-7} \mathrm{eV} / \mathrm{cm}^{3}$ provides the upper limit on the integral UHE neutrino flux $J_{v}(>E)$ (sum of all flavors). It is given 


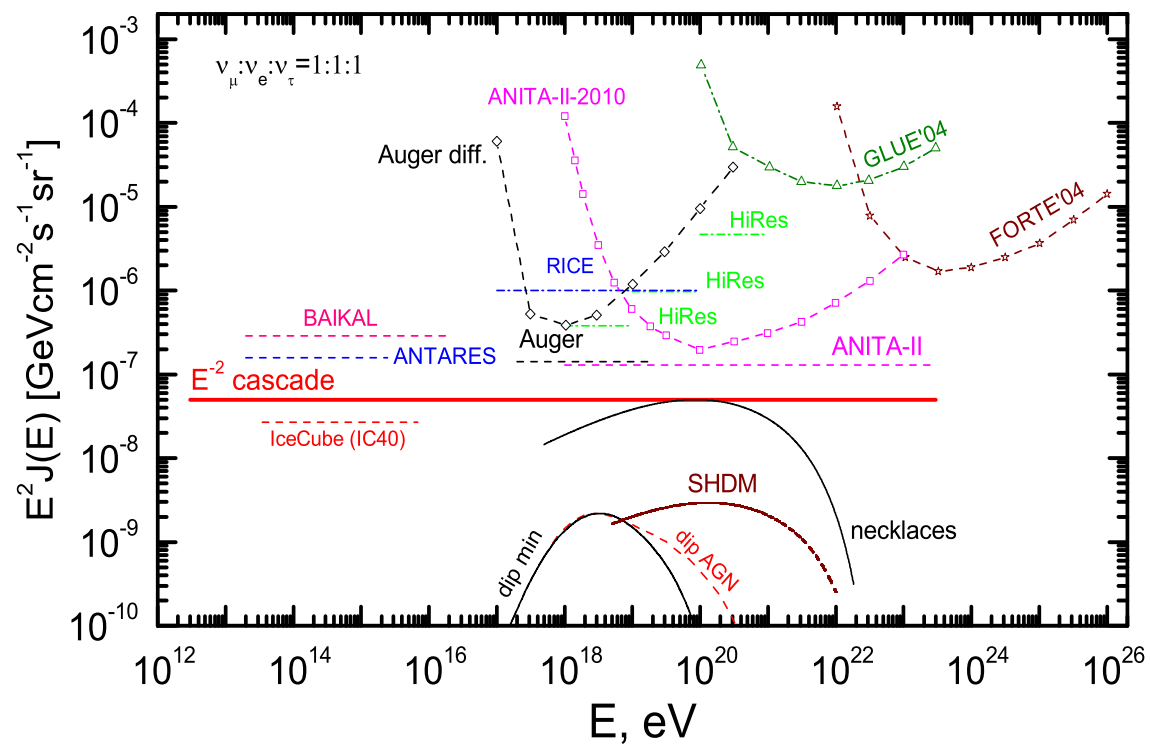

Figure 1: The experimental upper limits on UHE neutrino fluxes in comparison with e-m cascade upper limit in assumption of $E^{-2}$ generation spectrum (curve $E^{-2}$ cascade). Also shown are predictions for cosmogenic neutrinos in the dip model (curves dip-min and dip-AGN), for neutrinos from necklaces and from SHDM. Neutrino fluxes from necklaces and SHDM are normalized by AGASA data, and for normalization by HiRes data the fluxes should be diminished by factor $3-5$. Neutrino flux from superconducting strings is given by $E^{2} J(E)=$ const and it can reach the upper limit ' $E^{-2}$ cascade'. Neutrino fluxes are given for three flavors.

by the chain of the following inequalities

$$
\omega_{\mathrm{cas}}>\frac{4 \pi}{c} \int_{E}^{\infty} E J_{v}(E) d E>\frac{4 \pi}{c} E \int_{E}^{\infty} J_{v}(E) d E \equiv \frac{4 \pi}{c} E J_{v}(>E),
$$

where $\omega_{\text {cas }}<\omega_{\text {cas }}^{\max }$. Thus, Eq. (2.1) gives the upper limit on the integral neutrino flux, which can be expressed in terms of the upper limit on differential neutrino spectrum $J_{v}(E)$ as

$$
E^{2} J_{V}(E)<\frac{c}{4 \pi} \omega_{\text {cas }}^{\max }
$$

Eq. (2.2) gives the rigorous upper limit on the neutrino flux. It is valid for neutrinos produced by HE protons, by topological defects, by annihilation and decays of superheavy particles, i.e. in all cases when neutrinos are produced through decay of pions and kaons. It holds for arbitrary neutrino spectrum falling down with energy. If one assumes some specific shape of neutrino spectrum, the cascade limit becomes stronger. For $E^{-2}$ generation spectrum, which is used for analysis of observational data one obtains the stronger upper limit. Given for three neutrino flavors it reads

$$
E^{2} J_{v}(E) \leq \frac{c}{4 \pi} \frac{\omega_{\mathrm{cax}}^{\max }}{\ln \left(E_{\max } / E_{\min }\right)},
$$

This upper limit is shown in Fig. 1.

The most interesting energy range in Fig. 1 corresponds to $E_{v}>10^{21} \mathrm{eV}$, where acceleration cannot provide protons with energies sufficient for production of these neutrinos. At present the region of $E_{v}>10^{21} \mathrm{eV}$, and $E_{v} \gg 10^{21} \mathrm{eV}$, can be considered as a signature of top-down models, which provide these energies quite naturally. 
As one can see from Fig. 1 the present observational upper limit reached by IceCube with 40 strings is below the cascade upper limit. Crossing it, this detector enters the physically allowed region of neutrino fluxes, and it can be regarded as historical event. The WB upper limit is not relevant for UHE neutrinos: it is not valid for top-down scenarios because proton production is strongly suppressed for top-down sources, and it is very uncertain for cosmogenic neutrinos, where for the same proton flux the fluxes of accompanying neutrinos may differ by one-two orders of magnitudes (see Fig. 2). However, the WB upper bound remains the convenient low-flux benchmark for detection of neutrino fluxes.
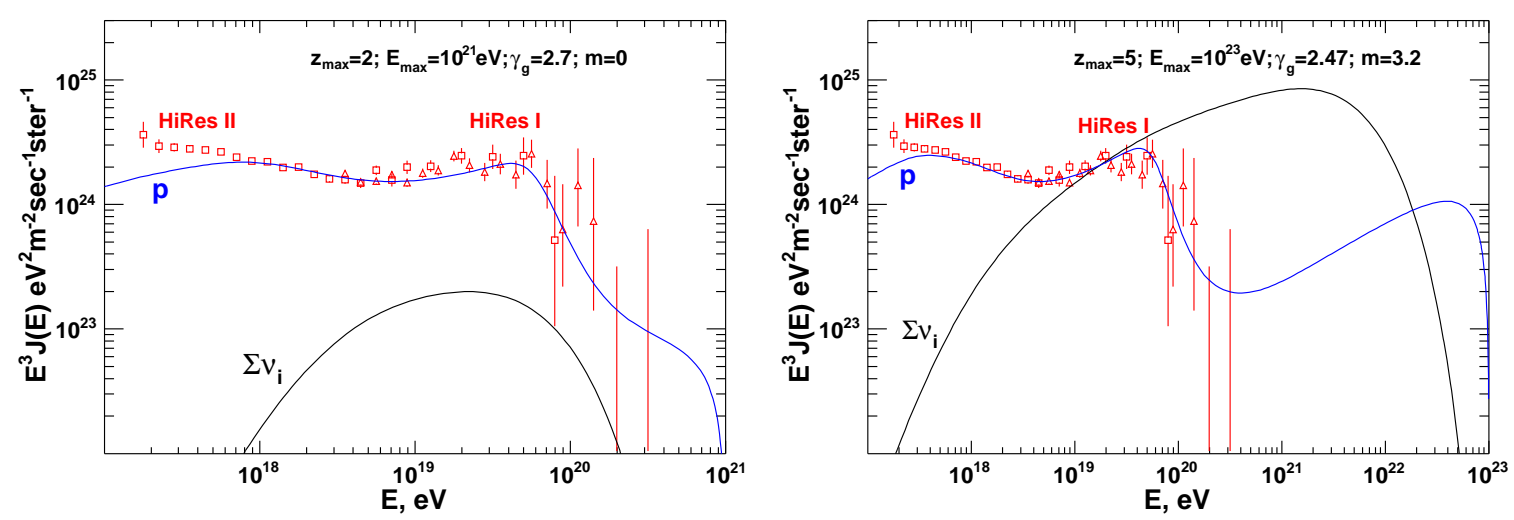

Figure 2: Diffuse neutrino fluxes in the dip models. Left panel: Non-evolutionary model and low $E_{\max }$. Neutrino flux is low: At all energies it is below the parent proton flux. Right panel: Evolutionary model and high $E_{\max }$. Neutrino flux is high: It can be higher than parent proton flux by order of magnitude. Note that in both cases the proton flux describes with good precision the HiRes spectrum.

\section{Cosmogenic neutrinos}

The main channel for cosmogenic neutrino production is interaction of UHE protons with CMB photons [5]-[13]. Two other channels of production are given by proton interaction with EBL photons (IR, optics, UV) and decay of neutrons (see [23] and references therein). These channels provide neutrino fluxes at energies lower than those from interaction with CMB. Production of neutrinos by UHECR nuclei are suppressed in comparison with protons (see for example recent calculations $[12,13])$. Therefore, the neutrino fluxes depend crucially on the mass composition of UHECR.

At present there is a dramatic conflict between recent observational data of two largest UHECR detectors, HiRes [24] and Auger [25]. While HiRes data evidence in favor of pure proton composition starting from energy $1 \times 10^{18} \mathrm{eV}$, the Auger data show the nuclei mass composition, getting progressively heavier in the range $(4-30) \times 10^{18} \mathrm{eV}$.

To maximise the neutrino flux we shall assume here the proton composition, normalizing the calculated flux by HiRes data. The HiRes energy spectrum show the presence of two features, pair-production dip and the GZK cutoff, both of which are the signatures of proton interaction with CMB photons. The GZK cutoff is well confirmed in the differential spectrum and in integral 
spectrum. In the latter the measured GZK characteristic $E_{1 / 2}=10^{19.73 \pm 0.07} \mathrm{eV}$ coincides well with the theoretical prediction [26] $E_{1 / 2}=10^{19.72} \mathrm{eV}$. The dip is a feature in the UHE proton spectrum, produced by $p+\gamma_{\mathrm{cmb}} \rightarrow p+e^{-}+e^{+}$[27], [28], [29]. The predicted dip is seen in the HiRes spectrum with $\chi^{2} /$ d.o.f. $=19.5 / 19$ [28]. In Figs. 2 and 3 one may see the good agreement of

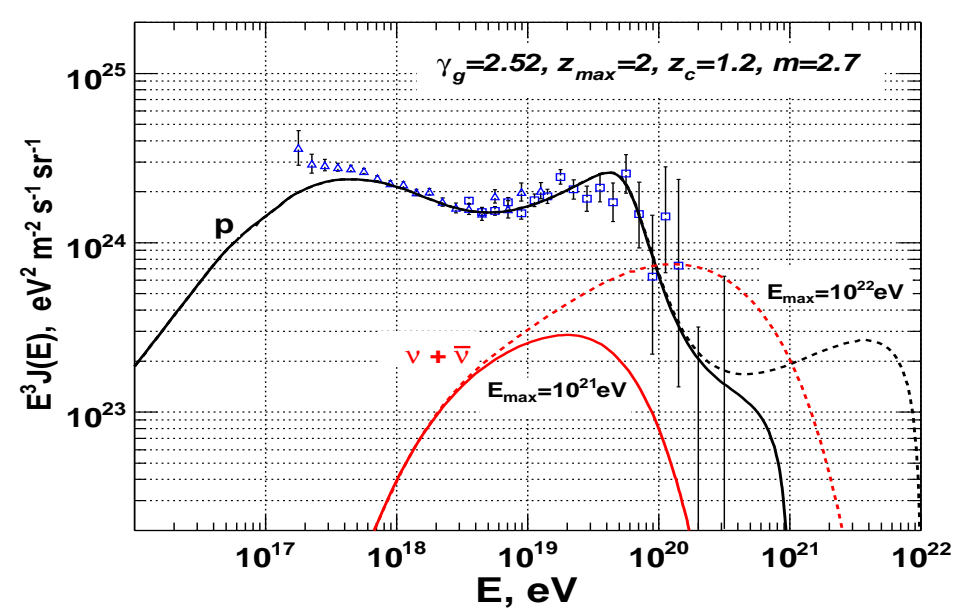

Figure 3: UHE neutrino flux in the dip model with AGN as the sources of UHECR. The cosmological evolution of AGN with $m=2.7$ up to $z_{c}=1.2$ is taken from X-ray observations of AGN. At larger $z$ the evolution is frozen up to $z_{\max }=2.0$. The fit of the dip is very good, though requires $\gamma_{g}=2.52$ different from the non-evolutionary case $m=0$. The neutrino fluxes are given here for one neutrino flavour.

the proton spectrum with HiRes data for three dip models: Non-evolutionary model (left panel of Fig. 2), dip model with a strong evolution (right panel of Fig. 2), and the realistic dip model (Fig. 3) with AGN as the sources [30], where AGN evolution is taken according to X-ray observations [31]. One may notice that the theoretical dip automatically describes the ankle observed at $E \approx$ $5 \times 10^{18} \mathrm{eV}$.

We shall present here the UHE diffuse neutrino fluxes calculated in the different versions of the dip models, most notably with cosmological evolution of the sources and without it, and using the different values of maximum acceleration energy $E_{\max }$. In all evaluations of neutrino spectrum we fit the HiRes data by the calculated spectrum of the protons.

To calculate neutrino flux produced by UHE protons it is enough to know the generation rate of UHE protons at each cosmological epoch. Wee take it as $Q(E)(1+z)^{m}$, where $Q(E) \propto E^{-\gamma_{s}}$, and factor $(1+z)^{m}$ describes the cosmological evolution of the sources up to some maximal redshift $z_{\max }$. In calculations of the proton spectrum we consider two cases:one without evolution, when we have only one free parameter, the generation index $\gamma_{g}$, and evolutionary scenario with three free parameters $\gamma_{g}, m$ and $z_{\max }$. The aim is to fit the observed HiRes spectrum with one calculated at $z=0$. In non-evolutionary scenario the best fit with very good $\chi^{2}$ is given by $\gamma_{g}=2.7$ (see Fig. 2 the left panel). Neutrino flux is low, less than the parent proton flux. However, the dip model with strong evolution and large $E_{\max }$, shown in the right panel, is characterised by large neutrino flux. The proton flux in this model fits the HiRes spectrum as good as non-evolutionary model.

In the realistic dip model one may assume [30] AGN as the sources and take the AGN evolution from X-ray observations [31]: $(1+z)^{m}$ with $m=2.7$ up to $z_{c}=1.2$, and frozen evolution from $z_{c}$ to 
$z_{\max }=2$. The generation index is fixed as $\gamma_{g}=2.52$ for the best fit of HiRes data (see Fig. 3). The calculated one-flavour neutrino fluxes for this model are shown in Fig. 3 for two values of $E_{\max }$.

\subsection{Cosmological evolution}

From Figs. 2 and 3 we see an impressive increase of neutrino flux due to cosmological evolution. Here we estimate analytically the evolution factor $k_{\mathrm{ev}}$. We demonstrate below that in most important UHE regime this evolution factor depends on evolutionary parameters $m$ and $z_{\max }$, and on generation index $\gamma_{g}$, but does not depend on neutrino energy $E$.

Neutrino number density at $z=0$ is calculated using neutrino generation rate $Q_{v}\left(E_{g}, z\right)$ :

$$
n_{v}^{\mathrm{ev}}(E)=\int_{0}^{z_{\max }} d z\left|\frac{d t}{d z}\right| Q_{v}\left(E_{g}, z\right) \frac{d E_{g}}{d E}
$$

where the explicit expressions for $|d t / d z|$ and $d E_{g} / d E$ are given in [28]. The protons with $E>$ $E_{\mathrm{GZK}}(z)$, responsible for neutrino production at epoch $z$, have very short life-time $\tau \ll H(z)^{-1}$ and produce neutrinos almost instantaneously. In this energy regime neutrino generation rate $Q_{v}\left(E_{g}, z\right)$ at any cosmological epoch $z$ has the same power-law generation index $\gamma_{g}$ as generation rate of primary protons:

$$
Q_{v}\left(E_{g}, z\right)=Q_{0}(1+z)^{m} E_{g}^{-\gamma_{g}},
$$

The observed neutrino energy $E$ is connected with generation energy at epoch $z$ as $E_{g}=(1+z) E$, and $d E_{g} / d E=1+z$. Using Eqs. (3.1) and (3.2) we obtain for evolutionary case:

$$
n_{v}^{\mathrm{ev}}(E)=\frac{Q_{0} E^{-\gamma_{g}}}{H_{0}} \int_{0}^{z_{\max }} d z \frac{(1+z)^{m-\gamma_{g}}}{\sqrt{\Omega_{m}(1+z)^{3}+\Omega_{\Lambda}}} .
$$

In the non-evolutionary case $n_{v}(E)$ is given by the equation above with $m=0$. The ratio $k_{\mathrm{ev}}$ of these two densities is plotted in Fig. 4. In the discussed energy regime it does not depend on $E$. The dependence of evolution factor on $m, z_{\max }$ and $\gamma_{g}$ is shown in Fig. 4.

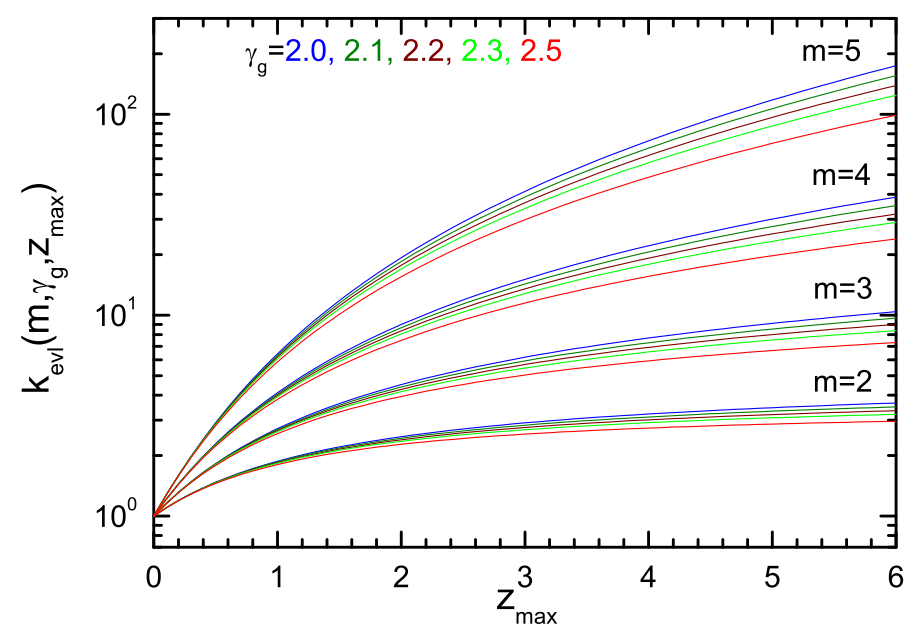

Figure 4: Cosmological evolution factor $k_{\mathrm{ev}}$ as function of $z_{\max }$ for different indices $\gamma_{g}$ and parameters of evolution $m$. The evolution factor $k_{\mathrm{ev}}$ is large for large $m$ and $z_{\max }$. 


\subsection{Minimum neutrino flux for pure proton composition}

We obtain now the minimum cosmogenic neutrino flux, assuming pure proton composition of UHECR. The procedure is shown in Fig. 5.

To obtain minimum neutrino flux we fix non-evolutionary model $m=0$ with small $z_{\max }=2$ and small $E_{\max }=1 \times 10^{21} \mathrm{eV}$. Choosing the smallest $\gamma_{g}=2.0$ compatible with energy convergence, we calculate the proton and neutrino spectra as shown by curves marked by 2.0 in Fig. 5. The proton spectrum is normalized by the HiRes flux at $E \approx 5 \times 10^{19} \mathrm{eV}$, i.e. at the beginning of the GZK cutoff. The produced neutrino spectrum is shown by black curve marked by 2.0. Now we gradually increase $\gamma_{g}$, which allows to fit the lower energy points in HiRes spectrum. The corresponding neutrino flux diminishes. At $\gamma_{g}=2.7$ we reach the energy point $7 \times 10^{17} \mathrm{eV}$ in the HiRes spectrum, and we are not allowed to increase $\gamma_{g}$ any more, because all available experimental data at lower energies favor the nuclei-dominated spectrum there. Therefore, the neutrino flux marked by the index $\gamma_{g}=2.7$ gives the minimum neutrino flux compatible with pure proton composition. This flux practically coincides with minimum dip neutrino flux shown in Fig. 1. This flux is undetectable by existing detectors and all projects, except maybe LORD [2].

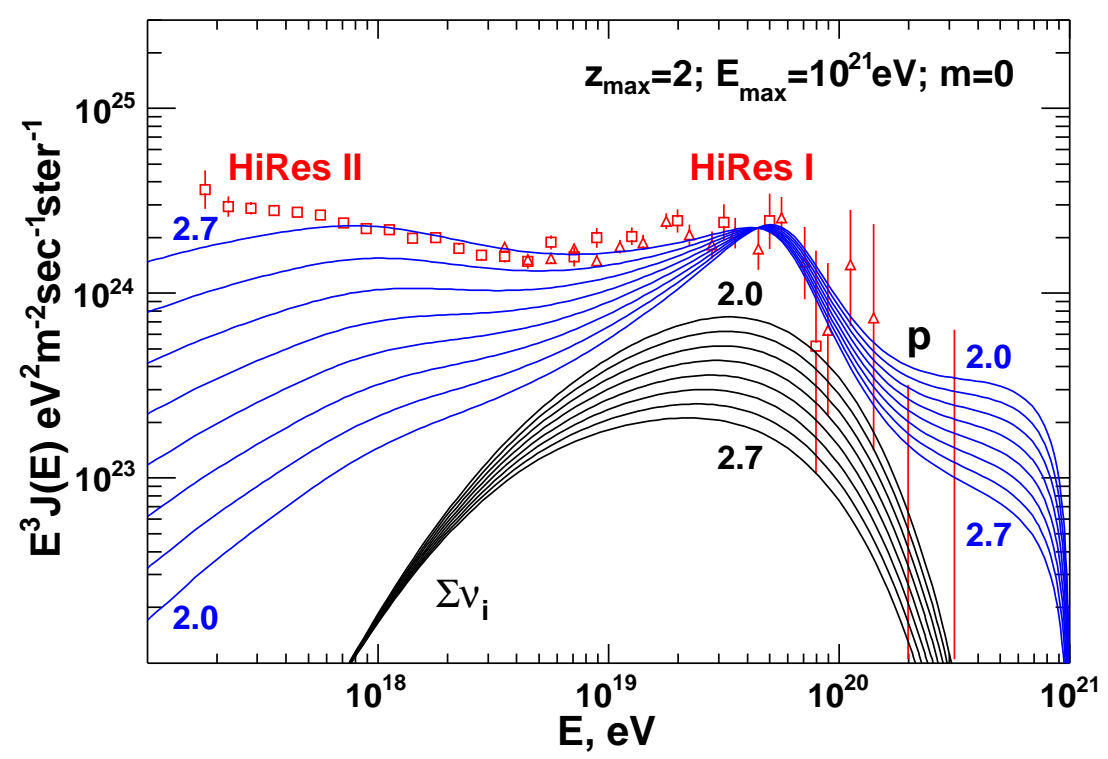

Figure 5: Minimum UHE neutrino flux (curve 2.7) for pure proton mass composition (see the text).

\section{Top-down models}

These sources include objects with annihilation of DM (the Sun, Earth, cores of the galaxies), objects with the decays of SHDM particles (galactic halos), TDs and mirror TDs. In the last three cases neutrinos are produced in the decays of superheavy particles with the masses up to $M_{\mathrm{GUT}} \sim 10^{16} \mathrm{GeV}$.

\subsection{Neutralino annihilation in the Sun and Earth}

- Neutralino is the best motivated DM particle. Crossing the Sun or Earth a neutralino can loose its energy in collisions with nuclei and diminish its velocity below the escape velocity. If 
it happens, a neutralino becomes gravitationally trapped in the object, and loosing further their energies, neutralinos are accumulated in the center of a celestial body [32]. Annihilating there they produce short-lived hadrons, e.g. D-mesons, which decay to neutrinos. The process of annihilation strongly depends on neutralino mass and composition (mixture of basic fields: zino, bino and two higgsinos).

These sources belong to the class of so called 'hidden sources', from which neutrinos are not accompanied by other radiations, most notably HE gamma radiation (see [17], [33], [34]).

\subsection{Superheavy Dark Matter (SHDM)}

The first proposal of SHDM [35] was motivated by Ultra High Energy Cosmic Rays (UHECR) and by natural character of DM production at the epochs soon after inflation. In particular SHDM particles can be produced gravitationally [36], when the Hubble parameter $H(t)$ exceeds the particle mass $H(t) \gtrsim m_{X}$. The observed density of DM in the universe $\Omega_{\mathrm{cdm}} \approx 0.23$, determines the mass of the particle as $m_{X} \sim 10^{13} \mathrm{GeV}$. The SHDM particles (X-particles) can be stable (due to e.g. discrete gauge symmetry) or quasi-stable (due to superweak discrete gauge symmetry breaking). The energy spectrum of produced particles has approximately power-law form at the highest energies $\propto E^{-1.9}$ [37]. The dominant decay particles are photons and neutrinos. As any cold DM, $\mathrm{X}$-particles are accumulated in the halos of galaxies, in particular in our galaxy with overdensity $2.1 \times 10^{5}$. One can expect the detectable fluxes of UHE photons and neutrinos from the Galactic Center region.

\section{Topological Defects (TDs)}

TDs are fundamental cosmological objects. They are produced in early universe due to symmetry breaking accompanied by the phase transitions. In many cases TDs become unstable and decompose to constituent fields, superheavy gauge and Higgs bosons (X-particles), which then decay producing UHE neutrinos (see $[14,15]$ for the reviews).

Ordinary strings.

Ordinary strings are one of the simplest TDs produced by $U(1)$ symmetry breaking. The main string parameter is the energy scale $\eta$ at which symmetry breaking occurs. Numerically $\eta$ is confined between EW mass scale $(\sim 100 \mathrm{GeV})$ and Grand Unified scale $\left(\sim 10^{16} \mathrm{GeV}\right)$. The width of a string is microscopically small $d \sim 1 / \eta$, the mass per unit length of a string $\mu$ is also determined by $\eta$ as $\mu \sim \eta^{2}$.

Inside string the symmetry is not broken, and all particles are massless. If there is some mechanism of exit outside (see below), such particle becomes massive due to symmetry breaking. The mass of the constituent field is always less than $\eta$, but the light particles in its zero-mass modes can inhabit a string. There are several mechanisms of particle exit from a string, which in the end results in production of UHE neutrinos: collapse of the string loops, intersection and self-intersection of the strings, production and annihilation of tiny loops. Below we discuss most efficient mechanism connected with cusp.

The strings exist in the form of long endless strings permeating the whole universe, and closed loops, formed at phase transition and due to intersections of long strings. At the moment of phase 
transition about $80 \%$ of the total string length is in the form of long strings and the remaining $20 \%$ is in closed loops. For a given scale $\eta$, taking into account shrinking of the loops due to gravitational radiation, the space density of the loops can be calculated.

Cusps.

Strings and string loops have the tremendous tension equal to mass per unit length $\mu$. Under action of this force a curved string and a closed loop are oscillating with relativistic speed. A fundamental property of a loop with such oscillation is existence of a particular point which obtains the light velocity $c$ each period. This point is called cusp. In the near-cusp region (segment) the points have distribution over the Lorentz factors. If the string particle exits through a segment with Lorentz factor $\Gamma$, its energy in the laboratory system is $E \sim \Gamma m_{X}$. Since in principle the Lorentz factor becomes arbitrary large approaching the cusp, and $m_{X}$ can be of GUT scale, the energy of emitting particle can exceed the Planck scale.

A jet with a large Lorentz factor $\Gamma$ determines the following properties of neutrino emission.

(i) Whatever are the angle distribution and energies of neutrinos in the frame of cusp, in laboratory system all neutrinos are propagating in the form of narrow jet with angle $\vartheta \sim 1 / \Gamma$.

(ii) The maximum energy of neutrinos in the frame of cusp $E_{v}^{\prime}$ is laboratory system is boosted by factor up to $2 \Gamma$.

(iii) The minimum energy of neutrinos (emitted in backward direction) is suppressed by Lorentz factor $\Gamma$, but flux of these neutrinos is negligibly low. The neutrinos emitted at small backward angles $\vartheta^{\prime}$ are energy-boosted by factor $P \Gamma$, where $P$ is a flux suppression, connected with small solid angle $\vartheta^{\prime}$ of emitted neutrinos.

An interesting model of emission of UHE particles by an ordinary string has been recently proposed by Vachaspati [38]. The SM Higgs interaction with string world-sheet can result in Higgs condensate on the string. The Higgses are emitted through cusp, producing a jet of decay particles boosted by the cusp Lorentz factor.

Superconducting strings

In a wide class of elementary particle models, strings behave like superconducting wires [39]. Moving through cosmic magnetic fields, such strings develop electric current. When the current reaches the critical value, the charge carriers escape from a string, turn into massive mode and decay. The current growth is strongly enhanced in the cusp segments due to their contractions. The energies of the particles are boosted by cusp Lorentz factor.

This scenario has been studied numerically in [40] with two main model features included. First, from all known structures of the universe, the excitation of electric current occurs most efficiently in clusters of galaxies where magnetic field reaches $B \sim 10^{-6} \mathrm{G}$ and filling factor $f \sim 10^{-3}$. Second, the symmetry breaking scale of order $10^{9}-10^{12} \mathrm{GeV}$ must be assumed for detectable neutrino fluxes. The typical Lorentz factor of the radiating cusp segment is calculated to be $\Gamma_{c} \sim 10^{12}$, and the maximum energy of emitted particle can reach $\Gamma_{c} \eta \sim 10^{22} \mathrm{GeV}$. The neutrino spectrum is assumed $\propto 1 / E^{2}$, similar to $\propto E^{-1.9}$ [37]. The spectrum $E^{2} J_{v}(E)=$ const can be very close to the $E^{-2}$-cascade upper limit in Fig.1.

Necklaces (monopoles connected by string).

These TDs are produced in the $G \rightarrow H \times U(1) \rightarrow H \times Z_{2}$ sequence of symmetry breaking, with 


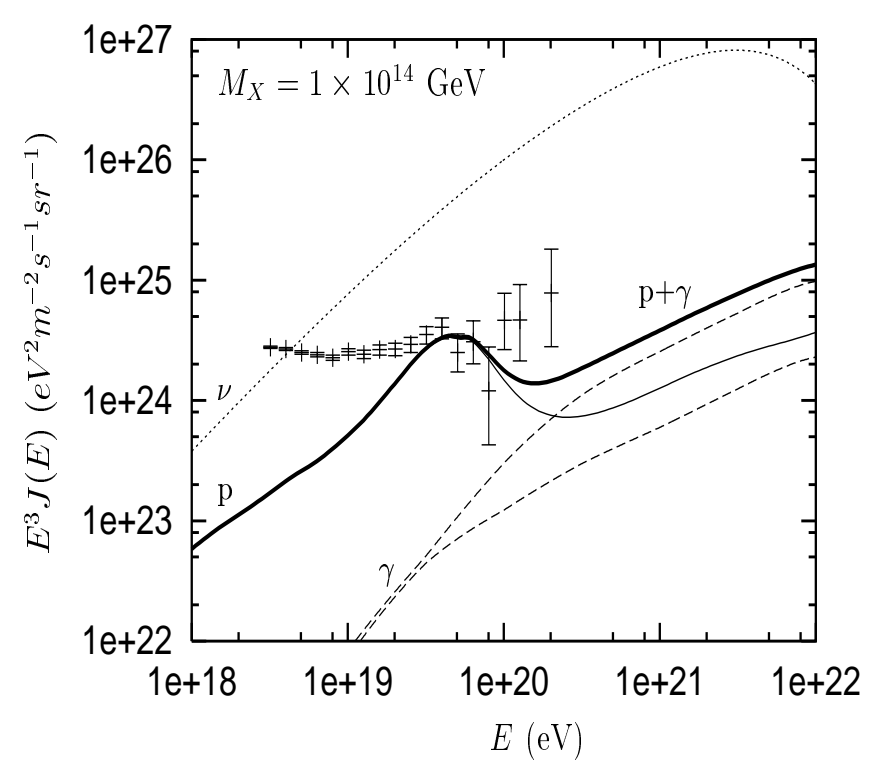

Figure 6: Diffuse all-flavour neutrino spectrum from necklaces for $m_{X}=1 \times 10^{14} \mathrm{GeV}$ [37]. The thick curve gives $p+\gamma$ flux normalized to the AGASA UHECR data. If to normalize the proton flux in this figure by HiRes data all curves, including one labelled $v$ (neutrino flux), should be lowered by factor 3 - 5 .

each monopole being attached to two strings, and a loop reminds a necklace with monopoles playing the role of beads. A scenario for UHECR and neutrino production has been proposed in [41]. In the process of evolution, the strings shrink due to gravitational radiation and $M \bar{M}$ pairs in the necklace inevitably annihilate, producing hadrons and neutrinos. Diffuse neutrino flux from necklaces are shown in Fig. 6 according to calculations in [37]. The fluxes in this figure are normalized by AGASA data. When normalised by HiRes data they are reduced by factor 3 - 5 .

\section{Mirror matter and mirror neutrinos.}

Mirror neutrinos give the only known example of fluxes not limited by the cascade upper limit. The concept of mirror matter, as first suggested by Lee and Yang [42], consists in existence of sector of matter fully symmetric with ordinary one and generated by space-reflection transformation. Kobzarev, Okun and Pomeranchuk [43] added the basic assumption that these two sectors communicate only gravitationally. The gravitational interaction results in mixing of mirror and ordinary neutrinos and their oscillations [44]. In two-inflaton cosmological model [45] the mirror matter is suppressed, while mirror TDs can strongly dominate. Mirror TDs copiously produce mirror neutrinos with extremely high energies. They oscillate into visible neutrinos, while all other mirror particles, which accompany production of mirror neutrinos, remain invisible for our detectors. Therefore, the upper limits on HE neutrinos in our world do not exist and their fluxes can be above the upper limit shown in Fig. 1. Neutrinos from TDs typically have very high energies and one can see that fluxes of discussed neutrinos are very severely constrained by ANITA-lite data [3].

\section{Conclusions}

The diffuse UHE neutrino radiation is presented here as cosmogenic and top-down neutrinos, in particular from Topological Defects (TDs). The fundamental problem of astrophysics involved 
in cosmogenic neutrinos is acceleration of particles. The shock acceleration at present knowledge of its theory cannot provide $E_{\max }$ higher than $10^{21}-10^{22} \mathrm{eV}$, and thus energies of cosmogenic neutrinos cannot exceed $3 \times 10^{20} \mathrm{eV}$. TDs naturally produce neutrinos emitted from cusps with energies by many orders of magnitude higher. Detection of neutrinos with these energies is a signature of top-down models.

Cascade upper limit is very general bound valid for both cosmogenic and top-down neutrinos. This upper limit became stronger with new Fermi-LAT data on extragalactic HE diffuse gammaradiation. From all existing detectors only IceCube reached the sensitivity below the cascade upper limit (see Fig. 1) and entered the physically allowed region for detectable neutrino fluxes. It can be considered as historical event for HE neutrino astronomy.

The flux of cosmogenic neutrinos can be large only in case UHECR are proton-dominated. Even in this case the flux is detectable if maximum acceleration energy $E_{\max }$ is large and sources have strong cosmological evolution (see [21, 22]).

Cosmogenic neutrinos of highest energies are detectable by future experiment JEM-EUSO in rather extreme models with large $E_{\max }$ and strong cosmological evolution (see [21, 22]).

The search for UHE neutrinos in any case is a search for a new physics, either for astrophysics (the new acceleration mechanisms and cosmological evolution of the sources) or for topological defects, mirror topological defects and superheavy dark matter.

\section{Acknowledgments}

I am grateful to Askhat Gazizov for many discussions and help in preparing the figures.

\section{References}

[1] Y. Takahashi (for JEM-EUSO collaboration) New J. Phys. 11 (2009) 065009.

[2] G. A. Gusev et al., Nucl. Instr. Methods A 604 (2009) S124.

[3] P. W. Gorham et al. Phys. Rev. D 82 (2010) 022004.

[4] K. Greisen, Phys. Rev. Lett. 16, (1966) 748, G. T. Zatsepin and V. A. Kuzmin, Pisma Zh. Experim. Theor. Phys. 4 (1966) 114.

[5] V. S. Berezinsky and G. T. Zatsepin, Phys. Lett. B 28 (1969) 423.

[6] R. Engel, D. Seckel and T. Stanev, Phys. Rev. D 64 (2001) 093010.

[7] O. E. Kalashev, V. A. Kuzmin, D. V. Semikoz, G. Sigl, Phys. Rev. D 66 (2002) 063004.

[8] Z. Fodor, S. Katz, A. Ringwald, H. Tu, JCAP 0311 (2003) 015.

[9] D. Hooper, A. Taylor, S. Sarkar, Astropart. Phys. 23 (2005) 11.

[10] M.Ave et al., Astropart. Phys. 23 (2005) 19.

[11] D. Seckel, T. Stanev, Phys. Rev. Lett. 95 (2005) 141101.

[12] D. Allard et al., JCAP 0609 (2006) 005.

[13] K. Kotera, D. Alard, A. V. Olinto, arXiv:1009.1382.

[14] P. Bhattacharjee and G. Sigl, Phys. Rep. 327 (2000) 109. 
[15] V. Berezinsky, Nucl. Phys. B (Proc. Suppl) 75A (1999) 119.

[16] V. Berezinsky and A. Smirnov, Ap. Sp. Sci. 32 (1975) 461.

[17] V. S. Berezinsky, S. V. Bulanov, V. A. Dogiel, V. L. Ginzburg and V. S. Ptuskin, Astrophysics of Cosmic Rays, North-Holland 1990.

[18] E. Waxman and J. Bahcall, Phys. Rev. D 59 (1999) 023002.

[19] P. Sreekumar et al., (EGRET collaboration) Astroph. J. 494 (1998) 523.

[20] A.A. Abdo et al., (Fermi-Lat collaboration), Phys. Rev. Lett. 104 (2010) 101101.

[21] V. Berezinsky, A. Gazizov, M. Kachelrieß, S. Ostapchenko, arXiv:1003.1496.

[22] M. Ahlers et al., Astropart. Phys. 34 (2010) 106; arXiv:1005.2620.

[23] T. Stanev, Nucl. Instrum. Meth., A 588 (2008) 215.

[24] R. U. Abbasi et al. (HiRes collaboration) Phys. Rev. Lett. 1002008101101.

[25] J. Abraham et. al. (Pierre Auger collaboration) Phys. Rev. Lett. 101 (2008) 061101,

[26] V. S. Berezinsky and S. I. Grigor'eva, Astron. Astrophys. 199 (1988) 1.

[27] V. Berezinsky, A. Z. Gazizov and S. I. Grigorieva, Phys. Lett. B 612 (2005) 147.

[28] V. Berezinsky, A. Z. Gazizov and S. I. Grigorieva, Phys. Rev. D 74 (2006) 043005.

[29] R. Aloisio et al., Astropart. Phys. 27 (2007) 76.

[30] V. Berezinsky, A. Gazizov, S. Grigorieva, arXiv:astro-ph/0210095.

[31] Y. Ueda et al., Astroph. J. 598 (2003) 886;

A. J. Barger et al., Astron. J., 129 (2005) 578.

[32] W. H. Press and D. N. Spergel, Ap.J 296 (1985) 679.

[33] V. Berezinsky, C. Castagnoli, P. Galeotti, Niovo Cim. C 8, (1985) 185.

[34] S. Razzaque, A.Yu. Smirnov, JHEP 03, (2010) 031.

[35] V. Berezinsky, M. Kahelriess, and A. Vilenkin, Phys. Rev. Lett. 79 (1997) 4302;

V. A. Kuzmin and V. A. Rubakov, Yad. Fiz. 61 (1998) 1122;

D. J. H. Chung, E. W. Kolb, and A. Riotto, Phys. Rev. D 60 (1999) 063504.

[36] D. J. H. Chung, E. W. Kolb, and A. Riotto, Phys. Rev. D 59 (1998) 023501;

V. Kuzmin and I. Tkachev, JETP Lett. 68 (1998) 271.

[37] R. Aloisio, V. Berezinsky, M. Kachelrieß, Phys. Rev. D 69, (2004) 094023.

[38] T. Vachaspati, arXiv:0911.2655.

[39] E. Witten, Nucl. Phys. B 249 (1985) 557.

[40] V. Berezinsky, K. Olum, E. Sabancilar and A. Vilenkin, Phys. Rev. D 80 (2009) 023014.

[41] V. Berezinsky, A. Vilenkin, Phys. Rev. Lett. 79 (1997) 5202.

[42] T. D. Lee and C. N. Yang, Phys. Rev. 104 (1956) 254.

[43] V. Kobzarev, L. Okun, I. Pomeranchuk, Sov. J. Nucl. Phys. 3 (1966) 837.

[44] Z. G. Berezhiani and R. N. Mohapatra, Phys. Rev. D 52 (1995) 6607,

V. Berezinsky, M. Narayan, and F. Vissani, Nucl. Phys. B 658 (2003) 254.

[45] V. Berezinsky and A. Vilenkin, Phys. Rev. D 62 (2000) 083512. 